\title{
3C: Pathological aspects of $H$. pylori infections
}

\section{C:01 RETINOBLASTOMA GENE PROTEIN EXPRESSION IN NON-MALIGNANT GASTRIC EPITHELIUM}

T.J. Havard, P. Sarsfield, H.W. Steer. Southampton General Hosp. Tremona Rd., Southampton

Using microwave antigen retrieval and an immunohistochemical technique we examined the density of Retinoblastoma gene protein $(\mathrm{RBp})$ expression and the proliferative state of gastric epithelium. The tissue samples were retrieved from the non-malignant resection margins of 24 gastrectomy specimens from patients with gastric adenocarcinoma.

The staining of the antibodies was quantified by cell counts and the severity of the inflammation in the specimens graded by the number of lymphoid follicles per $\mathrm{mm}$ of mucosa examined.

The epithelial cells of the proliferative compartment, around the neck of the crypts produced the densest staining of both antibodies. Little or no uptake of either antibody in the mature differentiated epithelial cells in the deep glandular or superficial lumenal areas.

There were two distinct levels of RBp expression. One group had a high level of $R B p$ staining and included all of the cases with severe inflammation. The cases with little or no inflammation had a lower level of $\mathrm{RBp}$ in the epithelium.

A similar relationship was found between the proliferative state of the epithelium and the RBp expression. The cell counts of the two antibodies (MiB-1 and RBp) in the proliferative compartment had a correlation coeficient 0.78 (95\% con. lim. $0.53-0.88$ ).

The role of mutational loss of function of RBp in the sequence of gastric tumour development and progression is as yet undefined. Our data provides strong evidence that in the hyperproliferative state associated with chronic gastritis RBp plays a vital role in the control of cell proliferation.

\section{C:02 DUODENAL GASTRIC METAPLASIA (GM) IN H. PYLORI (HP) POSITIVE PATIENTS WITH DUODENAL ULCER (UD) AFTER 5 YEARS HP ERADICATION OR AFTER H2 ANTAGONIST} MAINTENANCE TREATMENT

R. Suriani, E. Cerutti, D. Mazzucco, C. Pallante, M. Vajo, M. Ravizza, D. Dusio, G. Cera, E. Cardesi. Rivoli, Evangelico Valdese, Martini Hospitals Torino Italia

GM is considered an important pathogenetic factor in duodenal ulceration, but the results on whethever eradication of HP in UD patients reduces GM extension are conflicting.

Aim of the Work: To compare the presence of GM between a group of patients with UD and HP persistent eradication (mean \pm SD), 63 months \pm 14 and another of UD HP positive patients treated with $\mathrm{H} 2$ long term antagonist and to verify the occurrence of a significant GM regression. Subjects and Methods: We studied 16 asyntomatic patients (M:F 12:4), mean age $( \pm \mathrm{SD}) 54 \pm 12$, HP persistent negative on urea, Gram stain and Giemsa and with UD healed at scheduled serial endoscopies after triple antibiotic therapy performed in '89-'90. The control group, 13 symptomatic or asymptomatic patients (M:F 11:2) mean age ( \pm SD) 63 \pm 10 , consisted of patients HP positive on urea and/or Gram stain and/or Giemsa detection with UD diagnosed in the previous 5 years and in $\mathrm{H} 2$ antagonist maintenance therapy. Sections obtained from 3 duodenal biopsy speciments, were stained with diastase periodic acid -Schiff/Alcian blue and examined for GM. The GM extension was scored on a scale of 0-5. Results: $13 / 16(81 \%)$ of treated HP negative patients were GM positive and 7/13 (53\%) of HP positive control group patients were GM positive without statistical difference between the 2 groups $(P=0.11)$. Conclusion: Long term HP eradication does not reduce GM duodenal extension in UD healed patients.

\section{C:03 SHORT TERM HISTO-PATHOLOGICAL CHANGES IN PATIENTS WITH H. PYLORI INFECTION DURING TREATMENT WITH OMEPRAZOLE ALONE OR IN COMBINATION WITH AMOXICILLIN}

I. Adamsson, R. Seensalu, S. Sjöstedt, B. Wikström, C. Edlund, C.E. Nord. Karolinska Institute, Huddinge University Hospital, Stockholm, Sweden

We examined whether histo-pathological changes in the gastric mucosa during ongoing treatment could be used as an early marker to predict the outcome of $H$. pylori treatment.

Methods: 26 patients with dyspepsia submitted for upper endoscopy and with $H$. pylori infection were included in the study. The patients were randomised into two treatment groups: 13 patients received omeprazole capsules $20 \mathrm{mg}$ and amoxicillin capsules $1000 \mathrm{mg}$ twice daily for 14 days, and 13 patients received omeprazole $20 \mathrm{mg}$ and placebo twice daily for 14 days. Biopsies from corpus and antrum were collected before (day 0 ), during (day 10), and 4 weeks after end of treatment (day 42). H. pylor was detected with CLO, cultivation, PCR and histology. The biopsies from each location were also evaluated by an experienced pathologist according to the Sidney classification.

Results: The patients were divided into three groups: (A) $H$. pylori eradicated, treated with omeprazole and amoxicillin $(\mathrm{n}=7)$; (B) $H$. pylori not eradicated, treated with omeprazole and amoxicillin $(\mathrm{n}=6)$; (C) $H$. pylori not eradicated, treated with omeprazole and placebo $(n=13)$. In antrum, a significant decrease $(p<0.05)$ of inflammation and activity was noticed in all groups on day 10 . The scores for inflammation and activity remained low in group (A), tended to increase in group (B), and returned to pretreatment levels in group (C) on day 42 . No significant changes were found in the corpus mucosa in any of the groups. Also, no significant changes were seen in the extent of atrophy and metaplasia.

Conclusion: Early changes in the histological appearance during treatment do not predict $H$. pylori treatment outcome.

\section{C:04 MONOCLONAL ANTIBODY -BASED IMMUNO-FLUORESCENCE DETECTION OF H. PYLORI IN PARAFFIN SECTIONS OF GASTRIC TISSUE}

K. Pekkanen ${ }^{1}$, L. Loponen ${ }^{2}$, A. Pere ${ }^{2}$, Stig Nordling ${ }^{1} .{ }^{1}$ Helsinki University, Helsinki, Finland; ${ }^{2}$ Orion Diagnostica, Espoo, Finland

Purpose: The possibility of using monoclonal antibodies for detecting $H$. pylori in routine paraffin sections of gastric tissue was studied.

Materials and Methods: Indirect immunofluorescence staining (IFA) was used to test 17 monoclonal antibodies (MAb:s) for ability to stain $H$. pylori in paraffin sections of gastric tissue. The specificity of the MAb:s was assessed by IFA of bacterial strains. Direct immunofluorescence staining (FA), using one of the MAb:s, was done on 103 paraffin sections from 50 patients who's $H$. pylori status was unknown to the investigator. The FA results were compared with those of Giemsa staining done in routine pathology on sections from the same biopsies.

Results: Sixteen of the MAb:s were $H$. pylori -specific in IFA of bacterial strains. Nine of them stained $H$. pylori in IFA of gastric paraffin sections subjected to microwave treatment. Fourteen of the 50 patients were found to be $H$. pylori -positive by using FA. The concordance with the results of Giemsa staining was $100 \%$. The coccoidal forms of $H$. pylori, present in the sections from one patient, were also stained by the FA.

Conclusions: MAb-based FA is a powerful tool for detecting $H$. pylori in paraffin sections. Exposure of sections to microwaves prior to staining was necessary to obtain positive results with IFA and FA. The total slide handling time was shorter for Giemsa staining than for FA. However, the specificity, the easy interpretation of the results, and the possibility specifically to identify also the coccoidal forms of $H$. pylori, make FA a good alternative, at least for research purposes. 
3C:05 PREVALENCE OF HELICOBACTER HEILMANNII-LIKE ORGANISMS AND ASSOCIATED HISTOLOGICAL CHANGES OF THE HUMAN GASTRIC BIOPSIES. A PROSPECTIVE STUDY INCLUDING ONE CULTURE-CONFIRMED CASE

S. Holck ' , P. Ingeholm ${ }^{1}$, J. Blom ${ }^{2}$, A. Nørgaard ${ }^{3}$, L.P. Andersen ${ }^{3}$, L. Elsborg ${ }^{4}$, S. Adamsen $5 .{ }^{1}$ Depart of Pathol, Hillerød Hospital, Hillerød, Denmark; ${ }^{4}$ Dept. of Int Med, ${ }^{5}$ Dept. of Surg, Hillerød Hospital, Hillerød; ${ }^{2}$ Dept. of Molecular Cell Biol, Statens Serum Institut, ${ }^{3}$ Dept. of Clin Microbiol, University Hospital, Rigshospitalet, Copenhagen, Denmark

Aims: To determine the prevalence of $H$ heilmannii-like organisms $(H h)$ in human gastric biopsies and the associated histology compared with that of $\mathrm{H}$. pylori $(\mathrm{Hp})$-bearing gastric biopsies. Furthermore, the feasibility of culturing $H h$ was examined.

Methods: A consecutive series of 727 gastric biopsies from $650 \mathrm{pa-}$ tients were prospectively scrutinized for $H h$. Their distribution pattern was recorded as well as the affiliated morphology of the gastric mucosa. Additional biopsies from some of the patients were examined microbiologically.

Results: Four cases $(0.6 \%, 95 \%$ CI: $0.01-1.2 \%)$ of the examined material harboured $H h$. The bacterial burden was graded as sparse in three cases, moderate in one case. The distribution pattern was patchy; thus in no case did all biopsies from one endoscopy comprise $H h$. Adhesion to epithelial cells was infrequent. A mild gastritis, active in three cases, characterized all biopsies. Lymphoid aggregates occurred in biopsies from three patients. Micropapillary tufting of the epithelial layer and intestinal metaplasia were inapparent. Culture studies proved successful in the one of the four cases assayed.

Conclusions: The morphology of $H h$-bearing mucosa deviates from that of the $H p$-associated mucosa by the absence of epithelial damage of the former. This observation can in part be explained by the predominate location of $H h$ at a distance from the epithelium in contrast to the conspicuous $H p$-adhesion to epithelial cells, coupled with a usually low bacterial burden and patchy occurrence of $\mathrm{Hh}$ as opposed to the generally more heavy infestation with $H p$.

\section{C:06 THE SIZE OF LYMPHOCYTIC AGGREGATES IN CHRONIC GASTRITIS CORRELATED WITH THE HISTOLOGICAL IDENTIFICATION OF HELICOBACTER PYLORI}

P. Ingeholm ${ }^{1}$, S. Holck ${ }^{1}$, A. Nørgaard ${ }^{2}$, L.P. Andersen ${ }^{2}$, L. Elsborg ${ }^{3}$ ${ }^{1}$ Depart of Pathol, ${ }^{3}$ Depart of Int Med, Hillerød Hospital, Hillerød;

${ }^{2}$ Depart of Clin Microbiol, University Hospital, Rigshospitalet, Copenhagen, Denmark

Aim: To estimate the relationship between the size of lymphocytic aggregates (la) in cases of chronic gastritis and the $H$. pylori ( $H p$ ) status.

Methods: A consecutive series of 640 cases of chronic gastritis, serially sectioned and stained for hematoxylin-eosin, were scrutinized for $H p$. In selected cases Warthin-Starry-stained sections were likewise searched for $H p$. The presence of la, with or without germinal centres (gc), was recorded and their largest diameter was determined using a computer image analyser.

Results: Among 373 cases of $H p$-positive gastritis, 220 (59.0\%) comprised la, measuring 170-1160 $\mu \mathrm{m}$ (mean $381 \mu \mathrm{m}$ ); $52(25.7 \%)$ cases comprised gc. The material comprised 267 cases of $H p$-negative gastritis, 89 (33.3\%) of which harboured la, that measured 110-1900 $\mu$ m (mean 326 $\mu \mathrm{m})$. Gc were identified in $9(10.1 \%)$ of these cases.

Conclusion: The prevalence of la proved highest in $\mathrm{Hp}$-positive cases. It has here further been documented that the dimension of la in $\mathrm{Hp}$-positive cases tends to exceed that of la in $H p$-negative gastritis cases. The overlapping that does exist may in part be ascribed to the false negative cases that are known to occur when serological methods are not employed. Thus, the la-bearing, $H p$-negative gastritis cases may represent eradicated cases. The presence of gc proved highly suggestive of a $H p$-positive state. Hence, this study illustrates the likely evolution of la in relation to the gastric $H p$ status.

\section{C:07 HELICOBACTER PYLORI-INFECTED PATIENTS SHOW INCREASED INFLAMMATION IN GASTRIC BODY MUCOSA DURING LONG-TERM TREATMENT WITH LANSOPRAZOLE}

A.E. Berstad ' , J.G. Hatlebakk ${ }^{2}$, H. Maartmann-Moe ${ }^{2}$, A. Berstad ${ }^{2}$, P. Brandtzaeg ${ }^{1} .{ }^{1}$ Rikshospitalet, Oslo, Norway; ${ }^{2}$ Haukeland University Hospital, Bergen, Norway

Antral Helicobacter pylori infection and associated gastritis can spread proximally in the stomach during profound inhibition of acid secretion. Histological changes of the gastric body mucosa before and after long-term treatment with lansoprazole were examined. Methods: Four to six gastric biopsy specimens were taken from the major curvature $10 \mathrm{~cm}$ distal to the cardia from each of 110 patients ( 83 males; 27 females, median age
59 years) with reflux oesophagitis. After 12 weeks with administration of lansoprazole $(30 \mathrm{mg}), 103$ patients were endoscopically healed and asymptomatic and thereafter received maintenance therapy with lansoprazole (15 or $30 \mathrm{mg}$ ). Biopsy specimens obtained from the same location of the stomach before and after the treatment were available from each of 77 patients after 64 weeks (range, 12-77 weeks). Gastritis was graded according to the Sydney system. Results: Forty of 77 patients $(52 \%)$ were deemed to be infected with $\mathrm{H}$. pylori before and/or after therapy by immunohistochemistry. The median inflammation score had increased from grade 1 to grade 2 ( $p<0.001)$ in this group after treatment. Concomitantly, a higher activity score was observed in 28 of $40(p<0.001)$ patients, whereas a decrease was observed in only two patients. No moderate or high degree (grade 2 or 3 ) of atrophy was observed. Among the $H$. pylori-uninfected patients $(n=37)$, no significant change in grade of inflammation or activity was observed. Conclusions: A marked increase in body gastritis was observed in $H$. pylori-infected individuals during long-term therapy with the proton pump inhibitor lansoprazol, but no such change was observed in H. pylori-negative individuals.

\section{C:08 NO CHANGE IN INTESTINAL METAPLASIA AFTER H. PYLORI ERADICATION}

D. Fossati, C. Alvisi, R. Frego, M. Perego, O. Luinetti ${ }^{1}$, R. Fiocca ${ }^{1}$. Department of Internal Medicine, University of Pavia, IRCCS Policlinico S. Matteo; ' Department of Pathology, University of Pavia, IRCCS Policlinico S. Matteo

Aim: atrophic gastritis and intestinal metaplasia (IM) may be long term consequences of H. pylori (HP) infection, however it is not clear whether bacterial eradication can reverse these lesions. We studied the change in inflammation, activity and IM in a series of patients who underwent eradication therapy. Method: biopsy specimens were taken in the antrum (3), incisura angularis (2) and corpus (2) from 83 patients who underwent upper endoscopy for dyspeptic symptoms and showed both HP infection and IM. All were treated with omeprazole $20 \mathrm{mg} \mathrm{BID}$ and amoxicillin $1 \mathrm{~g}$ TID for 15 days and re-evaluated at 6 months by histology. Inflammation and activity were scored $(0-3)$ in accordance with the Sidney system criteria, while IM extension was evaluated on Alcian blue-PAS stained slides and expressed semiquantitatively as percent of affected mucosa. Data were analysed by Wilcoxon matched-pairs signed-ranks test. Results: eradication was obtained in 58 patients (GROUP A) and failed in 25 patients (GROUP B). As shown in the table, a significant reduction in both inflammation and activity occurred at 6 months and was mostly evident $(p<0.0001)$ in successfully treated cases. On the contrary no significant change occurred in IM spread.

\begin{tabular}{|c|c|c|c|c|c|c|c|c|c|}
\hline \multirow[t]{2}{*}{ Group } & \multicolumn{3}{|c|}{ Inflammation* } & \multicolumn{3}{|c|}{ Activity* } & \multicolumn{3}{|c|}{ Metaplasia* } \\
\hline & A & I & C & A & I & C & A & I & $\mathrm{C}$ \\
\hline $\begin{array}{l}\text { A (Entry) } \\
\text { A (6 months) } \\
\text { B (Entry) } \\
\text { B (6 months) } \\
\end{array}$ & $\begin{array}{l}1.93 \\
1.01 \\
1.96 \\
1.6 \\
\end{array}$ & $\begin{array}{l}1.96 \\
1.03 \\
1.88 \\
1.8 \\
\end{array}$ & $\begin{array}{l}1.81 \\
0.98 \\
1.48 \\
1.32 \\
\end{array}$ & $\begin{array}{l}1.44 \\
0.03 \\
1.24 \\
1 \\
\end{array}$ & $\begin{array}{l}1.36 \\
0.05 \\
1.2 \\
0.92 \\
\end{array}$ & $\begin{array}{l}1.29 \\
0.05 \\
0.8 \\
0.44 \\
\end{array}$ & $\begin{array}{l}17.22 \\
13.65 \\
17.88 \\
18.8 \\
\end{array}$ & $\begin{array}{l}16.5 \\
16.6 \\
18.4 \\
14.68 \\
\end{array}$ & $\begin{array}{l}1.56 \\
1.46 \\
3.6 \\
0.96 \\
\end{array}$ \\
\hline
\end{tabular}

*Mean values; A: antrum, I: incisura angularis, C: corpus

Conclusion: Information about the long-term effect on IM should be provided by longer follow-up studies.

\section{C:09 INTEROBSERVER VARIATION IN THE ASSESSMENT OF $\boldsymbol{H}$.} PYLORI GASTRITIS

R. Fiocca ${ }^{1}$, L. Villani ${ }^{2}$, M. Cornaggia $^{3}$, C. Tinelli ${ }^{2}$, E. Solcia ${ }^{1}$

${ }^{1}$ University of Pavia, Italy; ${ }^{2}$ IRCCS Policlinico S. Matteo, Pavia, Italy; ${ }^{3}$ Varese Regional Hospital, Italy

Aims: To evaluate the agreement between observers in scoring chronic gastritis according to the Sydney/Houston system criteria.

Methods: 200 consecutive cases with both antral (2) and body (2) specimens were blindly evaluated by three pathologists, primarly interested in GI pathology and with $>5$ yrs experience in grading gastritis. Two (A and B) were from the same Institution and have been working together for more than $10 \mathrm{yrs}$. H. pylori density was evaluated on Giemsa stained slides while Alcian blue-PAS was used for intestinal metaplasia. A 4-point (0-3) scale was applied in accordance with the Sydney/Houston criteria for scoring mononuclear inflammation, activity, $\mathrm{H}$. pylori density, atrophy, and intestinal metaplasia. k-Statistic was applied and the Svanholm's benchmarks were used for categorizing the level of agreement $(<0.50=$ poor, between 0.50 and $0.75=$ good, $>0.75=$ excellent).

Results: the $\mathrm{k}$ values in a pairwise comparison of observers are shown in the table. The degree of agreement in assessing the $\mathrm{H}$. pylori status was absolute $(k=1)$ for A vs B and excellent $(k=0.89)$ for A-B vs C.

Conclusions: a) the excellent agreement of A vs B for most parameters 


\begin{tabular}{lllll}
\hline \multirow{4}{*}{} & & & & \\
\cline { 3 - 5 } & & $\mathrm{A}$ vs B & $\mathrm{A}$ vs C & $\mathrm{B}$ vs C \\
\hline Antrum & Inflammation & 0.82 & 0.51 & 0.49 \\
& Activity & 0.77 & 0.61 & 0.58 \\
& H. pylori & 0.86 & 0.64 & 0.62 \\
& Atrophy & 0.59 & 0.49 & 0.42 \\
& Intestinal metaplasia & 0.92 & 0.81 & 0.75 \\
Corpus & Inflammation & 0.73 & 0.52 & 0.56 \\
& Activity & 0.81 & 0.54 & 0.59 \\
& H. pylori & 0.88 & 0.64 & 0.61 \\
& Atrophy & 0.72 & 0.47 & 0.43 \\
& Intestinal metaplasia & 0.84 & 0.74 & 0.80 \\
\hline
\end{tabular}

stresses the importance of a common personal experience in grading gastritis and consequently makes very reliable comparative studies performed in the same center. b) The $\mathrm{k}$ values of A-B vs $\mathrm{C}$ were constantly lower but still acceptable. c) Higher levels of disagreement occurred in grading atrophy.

\section{C:10 WHEN (1), WHERE (2), AND WHY (3) H. PYLORI COLONIZES THE PROXIMAL DUODENUM?}

D. Pospai, C. Vissuzaine, M. Merrouche, Th. Vallot, I. Sobhani, G. Cadiot, M. Mignon. Bichat-C. Bernard Hospital, Paris, France

Gastric metaplasia in the proximal duodenum (DGM) appears to be pre-existent obligatory, independent condition for duodenal ulcer (DU) development. While $\mathrm{Hp}$ gastritis is associated in most cases with DU disease $\mathrm{Hp}$ is inconstantly found in the proximal duodenum. To establish duodenal $\mathrm{Hp}$ prevalence in DGM areas, its location and determinants of duodenal $\mathrm{Hp}$ colonization 176 pts with Hp gastritis were evaluated prospectively: active DU (ADU), healed DU (HDU), duodenal erosions (DE), non-ulcer dyspepsia (NUD) and asympomatic endoscopicaly normal controls (C). Methods: $\mathrm{Hp}$ was detected by histology and immunohistochemistry (antibody-DACO B0471) in multiple biopsies ( 3 antrum, 3 corpus, 4-8 quadrantic in 1st doudenum and 2-3 on DU margins or scars when present) and double CLO test ( 1 antrum and 1 corpus biopsies). PAS (DGM prevalence and extent [(as \% of total epithelial surface measured in biopsies)] and HPS (grading of gastritis and duodenitis) stains were performed. Results: A) Prevalence of DGM and duodenal $\mathrm{Hp}$ in Hp gastritis pts: table

\begin{tabular}{llllll}
\hline Hp gastritis pts & ADU & HDU & DE & NUD & C \\
& $n=54$ & $n=43$ & $n=20$ & $n=43$ & $n=16$ \\
\hline DGM+ve \% (n) & $100(54)$ & $72(31)$ & $60(12)$ & $25(11)$ & $6(1)$ \\
HP+veDGM \% (n) & $29(16)$ & $29(9)$ & $8(1)$ & $9(1)$ & 0 \\
\hline
\end{tabular}

B) Location. In all DU pts with Hp detected in duodenum, $\mathrm{Hp}$ was located on DGM areas harbouring the niches or scars (100\%) and concomitantly outside the lesion in $28 \%$ cases only. In $\mathrm{Hp}+\mathrm{ve}$ DGM areas, active duodenitis was present in $100 \%$ on ulcer margins, $80 \%$ in scars and $42 \%$ in extra ulcer or scar site. C) Determinants of the preferential duodenal Hp colonization: a) larger extent of Hp colonized DGM vs non colonized DGM p $=0.03$ median (range) \%: $80(70-100)$ vs $50(10-100)$ respectively; b) significantly higher $(\mathrm{p}<0.001)$ association with Hp pangastritis in duodenal Hp+ve (19/27) vs duodenal Hp-ve (12/84) pts. Conclusions: Hp colonization of DGM was rarely found (1) affecting preferentially some DGM areas (2) which will became potential "battleground" for an ulcer development (3) in incompletely known conditions.

\section{C:11 DUODENAL GASTRIC METAPLASIA AND HELICOBACTER PYLORI INFECTION: A CROSS-SECTIONAL STUDY ON 196 CONSECUTIVE DYSPEPTIC PATIENTS}

M. Neri, L. Artese, O. Pesa ${ }^{1}$, M.A. Pistoia, L. Lombardi, A. Spadaccini ${ }^{2}$, D. Susi ${ }^{3}$, M. Ricciuti, F. Laterza, F. Carbone, F. Cuccurullo. ' Ospedali di Penne, Universita' de L'Aquila, Chieti, Italy; ${ }^{2}$ Ospedali di Vasto, Universita' de L'Aquila, Chieti, Italy; ${ }^{3}$ Ospedali di Termoli; Universita' de L'Aquila, Chieti, Italy; Universita' "G. D'Annunzio", Chieti, Italy

Gastric metaplasia (GM) in the duodenum is considered a potential pathogenic factor for duodenal ulcer in Helicobacter pylori (Hp) positive patients; however, previous studies have reported a highly variable prevalence of GM in dyspeptic patients, with or without ulceration, ranging from $22 \%$ to $90 \%$. One possible cause of such differences might be attributable to a bias in the selection of patients undergoing duodenal biopsy sampling. Aims of the present study were to evaluate the prevalence of GM in the duodenum according to: i) the presence and the density of $\mathrm{Hp}$ in the gastric antrum; ii) the endoscopic diagnosis iii) the presence and extent of chronic and active duodenitis. Methods: 196 consecutive dyspeptic patients undergoing endoscopy for the first time were studied. In all patients antral biopsies were taken for quick-urease test, H\&E and Giemsa staining, and anti Hp IgG were assayed in serum. Patients were considered Hp negative when all tests were negative. At histology, Hp density in the antrum was graded from 0 to 3 according to the number of bacteria. At least four biopsies were taken from the duodenum, one from each quadrant and stained with H\&E, PAS-Alcian and Giemsa, ther latter to assess the presence of Hp on GM. GM and its colonization by Hp were graded from 0 to 3 according respectively to the extent of PAS-positivity and number of bacteria. Results: Prevalence of GM was not different between $\mathrm{Hp}+$ and $\mathrm{Hp}-$ patients $(52 \%$ vs. $33 \%, \mathrm{p}=$ n.s. $)$, but patients with duodenal ulcers showed an higher prevalence of $\mathrm{GM}$ vs. all other diagnosis $(86 \%$ vs. $45 \%, \mathrm{p}<0.001$ ). GM colonization by $\mathrm{Hp}$ was present in $41 \%$ of $\mathrm{Hp}$ positive patients, but no relationship was observed between density of $\mathrm{Hp}$ in the stomach and presence of GM or extent of its colonization. A positive correlation was observed between presence of active duodenitis and GM ( $p<0.001$ ), while no relationship was found between chronic duodenitis and GM. Summary: GM is associated with the presence of duodenal ulcer and active duodenitis; neither Hp positivity nor its density in the gastric antrum are related to the occurrence of $\mathrm{GM}$ and its colonization by $\mathrm{Hp}$. Conclusion: Hp infection of the gastric antrum per se is not a major determinant of the occurrence of GM.

\section{C:12 DISTRIBUTION OF CagA H. PYLORI STRAINS IN THE STOMACH AND RELATION WITH HISTOLOGY}

N. Figura ${ }^{1}$, Z. Xiang ${ }^{2}$, C. Vindigni ${ }^{1}$, C. Gennari ${ }^{1}$, A. Covacci ${ }^{2}$ ${ }^{1}$ University of Siena, Italy; ${ }^{2}$ Biocine, Siena, Italy

Patients with peptic ulcer or gastric cancer are more likely to be infected by Helicobacter pylori $(H P)$ strains which express the vacuolating toxin and the associated protein CagA. CagA ${ }^{+}$strains are endowed with increased inflammatory potential. We investigated the proportion of $\mathrm{cagA}^{+}$ $H P$ strains in the stomach of dyspeptic patients, and compared the ratios of $\mathrm{cagA}^{+}$colonies with the histological findings. Biopsies from antrum, body and fundus of each of 19 patients were cultured on selective blood-agar. Five to 20 colonies per area per patient were subcultured, replicated on nitrocellulose and hybridised with a 297 bp cagA probe (Covacci et al. PNAS 1993, 90: 5791). CagA expression of some cagA $A^{+}$and cagA $A^{-}$ colonies was tested by Western blot (WB). Serum IgG to CagA were investigated by WB. $H P$ was present in all gastric areas in 17 cases. A total of 747 colonies were examined for cagA. All colonies were cagA $A^{+}$ (no. $=88$ ) in 4 , and $\mathrm{cagA}^{-}$(no. $=87$ ) in 2 cases. In 13 patients $(68.4 \%$ ), cagA $^{+}$and cagA $A^{-}$organisms (no. $=572$ ) were present in the stomach at the same time. The expression of CagA agreed with the cagA possession. $\mathrm{cagA}^{+}$colonies from the corpus $(50 \%)$ were significantly more numerous than from fundus ( $39.6 \%, P=0.024 ; 45.7 \%$ from antrum). The percentages of $\mathrm{cagA}^{+}$strains from 12 areas with histological normal mucosa, 36 with $\mathrm{mild} /$ moderate chronic gastritis, and 8 with atrophy or intestinal metaplasia $(\mathrm{A} / \mathrm{IM})$ were $34.1,50.8$ and 75.0 , respectively $(P=0.0004$, gastritis $v s$ A/IM). 18 patients were CagA seropositive. Mixed cagA $A^{+} / \mathrm{cagA}^{-}$strain infections are common. cagA ${ }^{+} H P$ mucosal colonisation is a risk factor for the development of pre-cancerous lesions.

\section{C:13 CHEMILUMINESCENT IMAGING OF IL-8 IN THE GASTRIC MUCOSA IN H. PYLORI INFECTION}

J.E. Crabtree, P. Pasini ${ }^{~}$, F. Bazzoli ${ }^{1}$, J.I. Wyatt, S. Perry, I.J.D. Lindley ${ }^{2}$, E. Roda ${ }^{1}$, A. Roda ${ }^{1}$. St. James's Hospital, Leeds, UK $;{ }^{1}$ University of Bologna, Italy; ${ }^{2}$ Sandoz Research Institute, Vienna, Austria

Purpose. Previous immunofluorescence studies have demonstrated increased IL-8 in the gastric epithelium in $H$. pylori infection. In vitro IL-8 mRNA and IL-8 secretion by gastric epithelial cells is stimulated by CagA $+H$. pylori. This study investigates $\mathrm{IL}-8$ in vivo in patients infected with CagAt and CagA negative $H$. pylori strains using a novel immunochemiluminescence technique which allows quantitative image analysis.

Methods. Cryosections of antral biopsies $(n=24)$ were incubated sequentially with a monoclonal antibody to IL-8 or isotype specific control antibody and peroxidase-conjugated goat anti-mouse antibody. Following addition of substrate, bound antibodies were detected using a low light imaging luminograph. CagA status was determined serologically and/or by PCR of cultured isolates.

Results. A chemiluminescence signal for IL-8 was observed in the gastric epithelium of all patients confirming earlier results. In HP neg patients $(n=6)$ and those infected with CagA neg strains $(n=8)$, IL-8 was generally uniformly expressed within the epithelium. In contrast, in CagA+ patients $(n=10)$ there was marked variation in epithelial IL-8 positivity with focal areas of high positivity being located either in the gastric pits or on the surface epithelium. Low areas of IL-8 expression, possibly reflecting areas of epithelial erosions and damage previously documented to be association with CagA infection, were interspersed with areas of high IL-8 positivity. Within the lamina propria of some CagA+ patients a 
gradient of decreasing IL-8 chemiluminescence from the epithelium was observed implying matrix imprinting of basally secreted IL-8 following apical bacterial stimulation occurs in vivo.

Conclusions: Sensitive chemiluminescence techniques can detect inflammatory mediators such as $\mathbb{I L}-8$ in the gastric mucosa and identify strain-specific variations in expression.

\section{C:14 GASTRIC EPITHELIAL CELL KINETICS AND INFLAMMATORY CELL INFILTRATION IN H. PYLORI ANTRAL GASTRITIS: QUANTITATIVE COMPUTER ASSISTED IMAGE ANALYSIS}

A. Armuzzi, E. Iascone, G. Pignataro, M. Covino, A. Valenti, A. DeLuca, M. Anti, G. Gasbarrini. Catholic University, Rome, Italy

Enhanced gastric epithelial cell proliferation could be involved in gastric carcinogenesis. Acute and chronic inflammation seem to have a role in the deregulation of epithelial cell kinetics. Aim of our study was to correlate gastric epithelial cell proliferation in $H$. pylori $(H P)$ positive and negative gastritis with inflammatory cell infiltration in the lamina propria evaluated by means of computer assisted image analysis. Methods: antral biopsies taken from each of twenty-two dyspeptic patients were processed for BrdU immunostaining [cell proliferation expressed as Labeling Index (LI)], for histology and Giemsa stain. Several sections (stained with H\&E) were blindly assessed with $\times 25$ objective and $\times 1000$ final magnification by an image analyzer. For each section six microscopic fields were randomly selected. Mononuclear (MNC) cells were automatically counted with a microdensitometric software, while polymorphonuclear (PMN) cells were counted with a morphometric software. Statistical analysis was made by Spearman rank test. Results: ten patients were $H P$ positive and twelve were $H P$ negative. Epithelial cell proliferation in antral pits was increased in $H P$ positive with respect $H P$ negative gastritis [LI $=5.9 \pm 0.9$ vs. $3.7 \pm 0.4$, (p $<0.05)$ ]. Both MNC and PMN cell density were higher in $H P$ positive than in $H P$ negative gastritis $\left[\mathrm{MNC}\left(\right.\right.$ cells $\left./ \mathrm{mm}^{2}\right)=6183.1 \pm 195.2$ vs. $3478.0 \pm$ 281.9, $(\mathrm{p}<0.000001) ; \mathrm{PMN}\left(\right.$ cells $\left./ \mathrm{mm}^{2}\right)=316.7 \pm 45.6$ vs. $126.4 \pm 16.6$, ( $\mathrm{p}<0.001)$ ]. Both MNC and PMN cell density were significantly related with enhanced cell proliferation $(\mathrm{p}<0.05)$. Conclusions: HP increases gastric epithelial cell proliferation. Our quantitative data indicate that one of the possible pathogenetic mechanisms could be acute and chronic inflammation.

\section{C:15 CLINICAL APPLICATION OF GASTRIC HISTOLOGY TO MONITOR TREATMENT OF DUAL THERAPY IN H. PYLORI ERADICATION}

Hsiao-Bai Yang, Bor-Shyang Sheu, Ih-Jen Su, Chung-Ho Chien, Xi-Zhang Lin. National Cheng Kung University Hospital, Tainan, Taiwan

Aims: To evaluate the changes in gastric mucosa after dual therapy and to compare the pretreatment histologic difference between the successful and fail groups. Method: 145 dyspeptic patients with $H$. pylori infection were treated with 2-wk course of dual therapy (Amoxicillin $500 \mathrm{mg} q 6 \mathrm{~h} \&$ Omeprazole $20 \mathrm{mg}$ bid). The $H$. pylori density (grade $0-5$ ) and modified Sydney system of gastric histology were applied to three pairs of gastric biopsy from antrum, body \& high body near cardia before and 8 weeks after treatment. Results: The eradication rate is $73.5 \%$ and the result of histologic findings are listed as Table I \& II.

Table I: Histologic changes by dual therapy

\begin{tabular}{llll}
\hline Histologic score & Pretreatment & Posttreatment & $p$ value \\
\hline Acute activity & $2.0 \pm 1.6$ & $0.3 \pm 0.7$ & $<0.0001$ \\
Chronic inflammation & $6.1 \pm 1.6$ & $4.3 \pm 1.6$ & $<0.0001$ \\
H. pylori density & $7.3 \pm 3.4$ & $1.1 \pm 2.3$ & $<0.0001$ \\
Eosin. infiltration & $2.1 \pm 1.2$ & $0.7 \pm 1.0$ & $<0.0001$ \\
\hline
\end{tabular}

Table II: Histologic parameters relevant to therapeutic success

\begin{tabular}{lllc}
\hline Histologic score (pretreatment) & Success & Failure & $p$ value \\
Total cases & 106 & 39 & \\
\hline Age & $52.6 \pm 14.5$ & $45.6 \pm 16.7$ & 0.015 \\
H. pylori density & $6.53 \pm 3.29$ & $9.51 \pm 2.81$ & $<0.01$ \\
Acute activity & $1.97 \pm 1.58$ & $2.13 \pm 1.74$ & NS \\
Chronic inflammation & $5.98 \pm 1.55$ & $6.49 \pm 1.65$ & NS \\
Eosinophil infiltration & $2.13 \pm 1.19$ & $1.97 \pm 1.06$ & NS \\
Intestinal metaplasia & $17.4 \%$ & $23.1 \%$ & NS \\
Lymphoid follicle & $32.1 \%$ & $64.1 \%$ & $<0.01$ \\
Atrophy & $26.4 \%$ & $48.7 \%$ & $<0.01$ \\
\hline
\end{tabular}

Conclusion: The inflammatory activity and $H$. pylori density were significant reduced. Therapeutic failure was related to the high pretreatment density of $H$. pylori, and presence of lymphoid follicle \& mucosal atrophy.
3C:16 RELATIONSHIP OF MAST CELLS WITH OTHER INFLAMMATORY CELLS IN H. PYLORI-INFECTED GASTRIC MUCOSA

S. Nakajima, D.Y. Graham, R.M. Genta. VA Medical Center and Baylor College of Medicine, Houston, TX, USA

Background: Mast cells are related not only to hypersensitivity reactions, but also to inflammation. There are few studies on mast cells in the human stomach. Aim: To evaluate whether mast cells in the gastric mucosa are related with $\mathrm{Hp}$-gastritis. Methods: We examined gastric biopsy specimens from 30 patients and volunteers with or without $H p$ infection. Subjects were divided into 4 groups; $13 \mathrm{Hp}$-infected patients with chronic active gastritis, $6 \mathrm{Hp}$-infected patients with peptic ulcer, 6 subjects with a normal stomach and 7 subjects with $\mathrm{Hp}$-negative chronic inactive gastritis. Biopsy specimens were stained with anti-human mast cell tryptase for the evaluation of mast cells. Serially cut sections were also stained with Genta stain for the evaluation of $\mathrm{Hp}$ infection and inflammation. Mast cells were counted in the mucosa and epithelium and the other inflammatory parameters were evaluated semi-quantitatively. We examined the relationship between mast cell density and the intensity of the infiltration of polymorphonuclear cells and mononuclear cells. Results: Mast cell density in $H p$-infected gastritis and peptic ulcer patients was significantly increased both in the mucosa and in the epithelium compared with normal subjects. Mast cell density significantly correlated with the intensity of the infiltration of polymorphonuclear cells and mononuclear cells. Mast cell density significantly decreased after cure of $\mathrm{Hp}$ infection. Epithelial mast cells had the same tendency as those in the lamina propria. Conclusion: Increased mast cell density in the stomach may initiate and/or regulate inflammation, or modulate ulcer healing. Mucosal mast cells may also play a role in $H$. pylori-associated alterations in acid secretion. 